\title{
Fissuras lábio-palatais: estudo caso-controle
}

\author{
Oral clefts: \\ a case-control study
}

Leonor de Castro Monteiro Loffredo*, José Maria Pacheco de Souza**, João Yunes**, José Alberto de Souza Freitas $^{* * *}$, Wilza Carla Spiri***

\begin{abstract}
LOFFREDO, L. de C.M. et al. Fissuras lábio-palatais: estudo caso-controle. Rev. Saúde Pública, 28: 213 7, 1994. Realizou-se um estudo caso-controle com o propósito de se detectar possíveis fatores de risco para o aparecimento de fissuras orais. Foram objeto de análise: local de moradia da mãe nos quatro primeiros meses de gestação, poluição, aplicação de pesticida/herbicida na lavoura, doenças dos pais, doenças da mãe nos quatro primeiros meses de gestação, ingestão medicamentosa nesse período, hereditariedade, tabagismo, consumo de bebida alcoólica e exposição a raio- $X$. Foram aplicados formulários às mães referentes aos 450 casos, sendo 354 portadores de fissuras labiais ou lábio-palatais e 96 de fissuras palatinas, e às mães referentes aos 450 controles. Empregou-se análise multivariada e as variáveis hereditariedade $(R R=4,96)$, epilepsia na mãe $(R R=2,39)$ e ingestão de anti-inflamatório $(R R=2,59)$ foram consideradas fatores de risco para fissuras labiais ou lábio-palatais. As variáveis hereditariedade $(R R=2,82)$ e poluição $(R R=2,58)$ foram consideradas fatores de risco para fissuras palatinas.
\end{abstract}

Descritores: Lábio leporino, epidemiologia. Fissura palatina, epidemiologia. Fatores de risco.

\section{Introduçáo}

As fissuras lábio-palatais são anomalias congênitas e integram dois grupos distintos de nosologias, do ponto de vista etiológico $0^{34,45}$, a saber:

- Fissura de lábio (unilateral ou bilateral) ou fissura lábio-palatina, resultantes da falta de fusão dos processos nasais da proeminência frontal com o processo maxilar na sétima semana de desenvolvimento embrionário.

- Fissura palatina, resultante da falta de fusão, na linha mediana, dos processos bilaterais independentes do maxilar por volta da décima segunda semana de vida intra-uterina.

Estudo epidemiológico realizado por Fogh-

* Departamento de Odontologia Social, Faculdade de Odontologia de Araraquara da Universidade Estadual Paulista - Araraquara, SP - Brasil

* Departamento de Epidemiologia, Faculdade de Saúde Pública da Universidade de São Paulo - São Paulo, SP Brasil

*** Hospital de Pesquisa e Reabilitação de Lesões LábioPalatais de Bauru da Universidade de São Paulo - Bauru, SP - Brasil

Separatas/Reprints: L. de C.M. Loffredo - Rua Humaitá, 1680 - Caixa Postal 331 - 14801-903 Araraquara, SP - Brasil

Edição subvencionada pela FAPESP. Processo 94/0500-0.
Andersen em 1942, citado por $\operatorname{Saxén}^{36}$, verificou que descendentes de portadores de fissura de lábio ou lábio-palatina apresentavam frequência maior deste tipo de fissura; por outro lado, descendentes de portadores de fissura palatina apresentavam ocorrência maior de fissura palatina. Outro estudo ${ }^{10}$ observou que a hereditariedade poderia desempenhar papel importante no aparecimento da fissura de lábio ou lábiopalatina, enquanto fatores ambientais deveriam ser analisados no estudo da fissura palatina. A maioria dos casos de fissura têm padrão de herança multifatorial ${ }^{6,11,23}$, estando uma minoria relacionada a um gene mutante específico, aberração cromossômica ou agentes ambientais específicos ${ }^{11,45}$.

Fontes de dados da anomalia são hospitais e clínicas especializadas ${ }^{1,26}$, certidoes de nascimen$\operatorname{tos}^{14,15,16,24,26,46}$ e maternidades ${ }^{5,26,38}$. O primeiro pesquisador a se preocupar com a prevalência das fissuras foi Frobelius citado por Fogh-Andersen ${ }^{10}$, encontrando 118 casos entre 180.000 crianças $(0,7$ casos por mil crianças) em um hospital de St. Petersburg, durante o período de 1833 a 1864 . Vários estudos sobre a frequiência da anomalia em diferentes países mostraram que ela prevalecia entre mongóis, era rara entre negros e ocupava posição intermediária entre caucasóides, conforme Tabela 1.

Estudos das possíveis associações do aparecimento de fissura em relação ao peso ao nascer ${ }^{2,7}$, idade gestacional ${ }^{8}$, ingestão de drogas nos primeiros meses 
Tabela 1. Prevalência (P) de fissuras lábio-palatais segundo etnia, país e ano.

\begin{tabular}{|c|c|c|c|c|}
\hline Etnia & $\begin{array}{c}P \\
(\times 1000)\end{array}$ & Pais & Data & $\begin{array}{c}\text { Referência } \\
\text { Bibliográfica N² }\end{array}$ \\
\hline $\begin{array}{l}\text { Mongol } \\
\text { Caucasóide } \\
\text { Negróide }\end{array}$ & $\begin{array}{c}2,68 \\
2,06 \\
1,30 \\
2,00 \\
0,23 \cdot 0,71 \\
0,53 \\
0,37 \\
0,32\end{array}$ & $\begin{array}{c}\text { Japão } \\
\text { Japáo } \\
\text { Inglaterra } \\
\text { Dinamarca } \\
\text { E.U.A. } \\
\text { Jamaica } \\
\text { Nigéria } \\
\text { África do Sul }\end{array}$ & $\begin{array}{l}1958 \\
1982 \\
1953 \\
1971 \\
1963 \\
1965 \\
1981 \\
1985\end{array}$ & $\begin{array}{r}32 \\
31 \\
21 \\
11 \\
2 \\
25 \\
18 \\
27\end{array}$ \\
\hline
\end{tabular}

de gestação ${ }^{13,35,40}$ e variação sazonal ${ }^{12,19,30,44}$, procuraram contribuir para o entendimento do desenvolvimento facial para se detectar oaparecimentoda fissura.

No Brasil, Nagem Filho e col. ${ }^{29}$ realizaram levantamento de escolares de Bauru-SP, encontrando a prevalência de 1,54 por mil, Cândido observou a prevalência de 0,88 por mil nascidos vivos do Município de Porto Alegre-RS e no estudo de Souza e col. ${ }^{43}$ essa relação foi de 0,47 por mil nascidos vivos em maternidades dos Estados de São Paulo, Rio de Janeiro e Santa Catarina.

O presente estudo usa abordagem caso-controle para investigar fatores de risco em relação ao aparecimento de fissura. Estudou-se a possível associação entre fissuras orais e as variáveis: morar na zona rural (nos 4 primeiros meses de gestação), exposição à poluição* (nos 4 primeiros meses de gestação), aplicação de pesticida e/ou herbicida na lavoura pelos pais, história de parentesco entre os pais, história familiar de fissura, história de epilepsia nos pais, história de hanseníase nos pais, ocorrência de doenças na mãe (rubéola, hipertensão, convulsão e diabetes - nos 4 primeiros meses de gestação), ingestão de medicamentos pela mãe (anti-inflamatórios, anti-eméticos, anti-convulsivantes, anti-hipertensivos, anti-alérgicos, anovulatórios, complexos vitamínicos, analgésicos e antibióticos - nos quatro primeiros meses de gestação), exposição da mãe a raio-X (nos 4 primeiros meses de gestação) ou exposição dos pais a raio- $X$ (um ano antes da gestação da mãe), ocorrência do consumo de bebidaalcoólica pela mãe e hábito de fumar dos pais.

\section{Material e Método}

Realizou-se um estudo caso-controle, com 450 casos e 450 controles. Foi considerado "caso" o portador de fissura labial ou lábio-palatina e de fissura palatina, sem malformações associadas. Tomou-se

* Gás de escapamento dos automóveis a motor Diesel, fuligens de usinas de álcool, indústria automobilística, fumaças de caldeiras, resíduos de lavagens de tanques industriais lançados na água dos rios. para "controle" o não-portador de quaisquer anomalias, sendo casos e controles crianças menores de um ano. A fonte dos casos foi o Hospital de Pesquisa e Reabilitação de Lesões Lábio-Palatais de Bauru-SP e a fonte de controles incluiu hospitais e maternidades das cidades de Bauru, Araraquara e São Paulo, no Estado de São Paulo, e de Londrina, no Estado do Paraná.

As informações relativas às variáveis de análise foram obtidas por entrevistas junto às mães dos casos e controles, usando formulário estruturado pré-codificado. Considerando que as condições especiais para este tipo de problema de saúde levaram à escolha de casos num local e de controles em diferentes cidades e que a origem de vários casos era o Município de São Paulo, adotou-se "a mãe morar em São Paulo" como possível fator de modificação de efeito.

Uma primeira etapa foi estudar univariadamente os fatores de risco; para cada variável de análise construiu-se uma tabela $2 \times 2$ estudou-se a respectiva razão dos "odds" (RR) ${ }^{39}$ como indicador daassociação caso/exposição. A significância estatística foi avalia$\mathrm{da}^{3}$, adotando-se o nível de $5 \%$ para tomada de decisão e subseqüente entrada na análise multivariada.

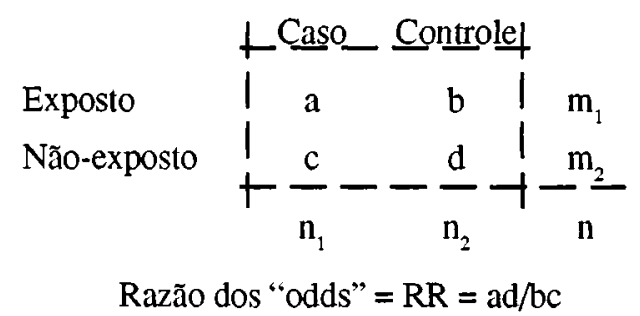

Foram selecionadas para comporem, em seguida, a análise multivariada as variáveis que satisfizessem os seguintes critérios:

- associação com significância estatística;

- $R R>2$ (ou $R R<0,5$ ), independentemente da significância estatística;

- RR em sentidos opostos, segundo local de moradia da mãe.

O modelo de análise multivariada foi o de regressão logística não-condicional ${ }^{37,42}$. Obteve-se as estimativas (RR), ajustadas para variáveis de confusão, 
por ponto e por intervalo de $95 \%$ de confiança, das razões de prevalência ao nascer, utilizando-se o programa MULTLR*. Estas razões de prevalência indicam que a presença do respectivo fator é um risco em relação ao aparecimento de fissura.

Decidiu-se analisar os dois tipos de fissura em separado, usando-se os mesmos controles.

\section{Resultados e Discussáo}

Entre os 450 controles e 450 casos estudados, $354(78,6 \%)$ apresentavam fissura labial ou lábiopalatina e $96(21,3 \%)$ fissura palatina. Esse achado vai ao encontro da maioria de trabalhos realizados, em queo tipo de fissura prevalente é a fissura labial ou lábiopalatina ${ }^{9,17,31}$, com exceção de pesquisa entre filandeses ${ }^{28}$.

Quanto à distribuição por sexo, verificou-se predomínio do sexo masculino entre portadores de fissura labial ou lábio-palatina, sendo que a fissura palatina é mais prevalente no sexo feminino, já tendo sido observadoesse comportamentoem vários trabalhos ${ }^{4,12,19,21,33}$.

\section{Fissura labial ou lábio-palatina:}

A análise univariada incluiu 354 casos e 450 controles, distribuídos segundo local de moradia da mãe - morar no Município de São Paulo (SP) ou morar fora do Município de São Paulo (NSP).

As variáveis hereditariedade, poluição e hipertensãoapresentaram riscos relativos significantes para moradores do Município de São Paulo, valendo, respectivamente, 20,20,4,20 e 3,27. Para moradores de fora do Município de São Paulo, as seguintes variáveis apresentaram-se como fator de risco: aplicação de pesticida e/ou herbicida na lavoura pela mãe ( $R R=6,12)$, hereditariedade ( $R R=3,93$ ), epilepsia na mãe $(R R=2,67)$, ingestão de anti-inflamatório $(\mathrm{RR}=1,84)$ e exposição da mãe a raio-X nos quatro primeiros meses de gestação $(R R=8,25)$.

Como o risco relativo (RR) foi indefinido em um dos estratos-SP, para as variáveis aplicação de pesticida e/ou herbicida na lavoura pela mãe e exposição a raio- $\mathrm{X}$ nos quatro primeiros meses de gestação, as mesmas foram eliminadas para compor a análise multivariada.

Por outro lado, inclui-se como variável morar em São Paulo (SP), bem como as interações que resultaram significantes estatisticamente: poluição e SP, hereditariedade e SP e hipertensão e SP.

$A$ análise multivariada identificou como fatores de risco para fissura labial ou lábio-palatina: hereditariedade, epilepsia na mãe e ingestão de anti-in-

Multlr User's Manual - Ludwing Institute For Cancer Research - São Paulo, 1988. flamatórios (Tabela 2).

Em relação à hereditariedade, observou-se que todos os casos de fissura labial ou lábio-palatina, que apresentaram antecedentes familiares de fissuras, referiram-se à história de fissura labial ou lábio-palatina e nunca de fissura palatina.

A importância da hereditariedade no aparecimento da fissura labial ou lábio-palatina é um consenso entre os estudiosos ${ }^{10,26}$. Melnick e col. ${ }^{23}$ referem-se à susceptibilidade genética para agentes ambientais, de forma que a exposição a esses agentes, em período crítico, levaria a um efeito teratogênico, se se tratasse de um ser geneticamente predisposto.

Os resultados prévios do efeito modificador que a variável morar em São Paulo exerceria, permitiu observar riscos relativos para hereditariedade de 4,96e 15,67 , respectivamente a NSP e SP, embora as interações tenham sido estatisticamente não-significantes.

Quanto à epilepsia na mãe, observou-se risco relativo de 2,39 , embora o intervalo de confiança estivesse no limite de significância. Essa variável tem apresentado resultados controvertidos, no que diz respeito à sua importância como fator de risco ou à ingestão de drogas anti-convulsivantes. Assim, Shapiro e col. ${ }^{39}$ e Bonaiti e col. ${ }^{4}$ ressaltam a associação da epilepsia à anomalia, enquanto outros estudiosos ${ }^{22,41}$ referem-se à importância do anti-convulsivante no aparecimento das fissuras. Algumas dificuldades surgem no estudo da relação entre epilepsia, droga anticonvulsivante e fissura, referentes ao número reduzido de grávidas epilepticas, baixa prevalência de fissuras e da distinção entre o papel da droga e da própria epilepsia; além disso, o tratamento pode incluir mais do que uma droga e a comparação entre tratamentos pode ser prejudicada pela diferença entre tipos de epilepsia.

Outro fator de risco para fissuras labiais ou lábiopalatais foi a ingestão de anti-inflamatório nos quatro primeiros meses de gestação. Fogh-Andersen" observa a necessidade de não ingerir quaisquer drogas nos quatro primeiros meses de gestação, excetuando-se os complexos vitamínicos. Nopresente estudo, a ingestão de complexo vitamínico resultou fator de proteção na análise multivariada $(R R=0,55)$.

\section{Fissura palatina:}

A análise univariada inclui 96 casos e 450 controles, distribuídos segundo local de moradia da mãe: morar no Município de São Paulo(SP) e morar fora do Município de São Paulo (NSP).

Para moradores de São Paulo, a poluição apresentou-se como fator de risco $(R R=10,00)$. Para moradores de fora de São Paulo, foram fatores de risco as variáveis ocorrência de raio- $X$ na mãe ( $R R=15,84)$, aplicação de pesticida e/ou herbicida 
Tabela 2. Fissura labial ou lábio-palatina. Estimativas por ponto (RR) e por intervalo (IC) de $95 \%$ de confiança da razão de prevalência para fatores de risco.

\begin{tabular}{lrc}
\hline Fator de Risco & RR & IC \\
\hline Hereditariedade(NSP) & 4,96 & $2,99 \cdot 8,22$ \\
(SP) & 15,67 & $4,01 \cdot 61,19$ \\
Epilepsia na mãe & 2,39 & $1,01 \cdot 5,69$ \\
Ingestão de droga anti-inflamatória & 2,59 & $1,35 \cdot 4,98$ \\
\hline
\end{tabular}

(NSP) : não morador de Sāo Paulo

(SP) : morador de São Paulo

Tabela 3. Fissura palatina. Estimativas por ponto (RR) e por intervalo (IC) de $95 \%$ de confiança da razăo de prevalência para fatores de risco

\begin{tabular}{clc}
\hline Fator de Risco & RR & IC \\
\hline Hereditariedade & 2,82 & $1,45 \cdot 5,50$ \\
Poluição (NSP) & 2,58 & $1,51 \cdot 4,41$ \\
(SP) & 9,42 & $2,34 \cdot 37,94$ \\
\hline
\end{tabular}

(NSP) : não morador de São Paulo

(SP) : morador de São Paulo

na lavoura pelo pai $(R R=7,38)$, poluição $(R R=2,43)$ e hereditariedade $(R R=2,22)$.

Conforme os critérios adotados para a seleção de variáveis para compor a análise multivariada, deveriam ser incluídas atividade da mãe na lavoura, aplicação de pesticida e/ou herbicida na lavoura pelo pai e ocorrência de raio-X na mãe nos quatro primeiros meses de gestação, porém, os respectivos riscos relativos foram indefinidos para um dos estratos-SP.

Algumas variáveis apresentaram risco relativo de grande magnitude, mas sem significância estatísti$\mathrm{ca}$, referentes à hipertensão-SP $(\mathrm{RR}=2,97)$, ingestão de anovulatório-SP $(R R=4,25)$, ingestão de complexo vitamínico-SP $(R R=4,86)$ e ingestão de drogas abortivas-SP $(R R=4,25)$.

Não foi possível analisar a variável hereditariedade para o estrato SP devido a problemas na inversão de matrizes.

Morar em São Paulo foi incluída como variável, bem como a interação poluição e São Paulo.

A análise multivariada detectou como fatores de risco para fissura palatina hereditariedade e poluição, conforme Tabela 3.

Em relação à hereditariedade, os portadores de fissura palatina que apresentam antecedente familiar de fissura, referiram-se à história de fissura palatina e nunca de fissura labial ou lábio-palatina.

Quanto à poluição, estudo experimental ${ }^{18} \mathrm{em}$ camundongos evidenciou essa variável como fator de risco, e Leck ${ }^{20}$ observou que a fissura palatina apresentava maior variabilidade do que a labial ou lábiopalatina em cada grupo da população da mesma etnia, sugerindo que o meio ambiente estivesse envolvido na sua etiologia.
Convém acrescentar que a abordagem genérica que foi dada à poluição pode ser levada a distorções na resposta do entrevistado.

LOFFREDO, L. de C.M. et al. [Oral clefts: a case-control study]. Rev. Saúde Pública, 28: 213-7, 1994. This study relates to a case-control analysis for the purpose of verifying the association between oral clefts and possible risk factors. The analysed variables were: place of mother's residence (urban/ rural), pollution, parental diseases, mother's diseases during the first four months of pregnancy, intake of drugs related to this period, heredity, smoking habits, alcohol consumption and $\mathrm{X}$-ray examinations during pregnancy or $\mathrm{X}$-ray examinations prior to preganacy. There were 450 cases of clefts of whom 354 had a cleft lip with or without cleft palate and 96 had a cleft palate. The relative risk (RR) for each variable by was estimated points and at a $95 \%$ of confidence interval and multivariate analysis was applied. As regards cleft lip with or without cleft palate, the risk factors are heredity $(R R=4.96)$, epilepsy in the mother $(R R=2.39)$ and the intake of drugs such as antiinflammatory substance in the first four months of pregnancy $(R R=2.59)$. Related to cleft palate, the risk factors are heredity $(\mathrm{RR}=2.82)$ and pollution $(\mathrm{RR}=2.58)$.

Keywords: Cleft lip, epidemiology. Cleft palate, epidemiology. Risk factors.

\section{Referências Bibliográficas}

1. ABYHOLM, F.E.Cleft lip and palate in Norway. I. Registration, incidence and early mortality of infants with CLP. Scand $J$. Plast. Reconstr. Surg, 12: 29-34, 1978.

2. ALTEMUS, L.A. The incidence of cleft lip and palate among North American negroes. Cleft palate J, 3: 357-61, 1966.

3. BERQUÓ, E. et al. Bioestatística. São Paulo, E.P.U., 1981.

4. BONAITI, C. et al. An epidemiological and genetic study of facial clefting in France. I. Epidemiology and frequency in relatives. J.Med. Genet.,19: 8-15, 1982. 
5. CÂNDIDO, I.T. Epidemiologia das fendas de lábio e/ou palato: estudo de recém-nascidos em dois hospitais de Porto Alegre, no período de 1970 a 1974. Porto Alegre, 1978. [Dissertação de Mestrado - Universidade Federal do Rio Grande do Sul].

6. CHUNG, C.S. et al. Segregation analysis of cleft lip with or without cleft palate: a comparison of Danish and Japanese Date. Am. J. Hum. Genet., 39: 603-11. 1986.

7. CONWAY. H \& WAGNER, K.J. Incidence of clefts in New York City. Cleft Palate J., 3: 384-90, 1966.

8. DONAHUE, R.F. Birth variables and the incidence of cleft palate: Part II. Cleft Palate J. 4: 234-9, 1967.

9. FOGH-ANDERSEN, P. Vital statistics of cleft lip and palate past, present, future. Acta Chir. Plast., 5:169-74, 1963.

10. FOGH-ANDERSEN,P. Genetic and non-genetic factors in the etiology of facial clefts. Scand J. Plast. Reconstr. Surg., 1: $22-9,1967$.

11. FRASER, F.C. The genetics of cleft lip and cleft palate (review) Am. J. Hum. Genet., 22: 336-52, 1970.

12. FRASER, F.C. Etiology of cleft lip and palate. In: Grabb, W.C. et al., ed. General aspects of clefts lip and palate. Boston, Little, Brown and Company, 1971. p. 54-65.

13. GOLDING, J. et al. Matemal anti-nauseants and clefts of lip and palate. Hum. Toxicol., 2: 63-73, 1983.

14. GREENE, J.C. et al. Epidemiologic study of cleft lip and cleft palate in four states. J. Amt. Dental. Assoc., 68: 73-90, 1964.

15. GREENE, J.C. et al. Utilization of birth certificates in epidemiologic studies of cleft lip and palate. Cleft PalateJ., 2: $141-56,1965$.

16. HAY, S. Incidence of clefts and parental age. Cleft Palate J., 4: 205-13, 1967

17. IREGBULEM, L.M. The incidence of cleft lip and palate in Nigeria. Cleft Palate J, 19: 201-5, 1982.

18. JANERICH, D.T.\& POLEDNAK, A.P. Epideniology of birth defects. Epidemiol. Rev., 5: 16-37, 1983.

19. KNOX, G. \& BRAITHWAITE, F. Cleft lips and palates in Northumberland and Durbam. Arch. Dis. Child., 38: 66-70, 1963.

20. LECK, I. The etiology of human malformations: insights from epidemiology. Teratology, 5: 303-14, 1972.

21. MAC MAHON, B. \& MCKEOWN, T. The incidence of harelip and cleft palate related to birth rank and maternal age. $A m$. J. Hum. Genet., 5: 176-83, 1953.

22. MEADOW, S.R. Congenital abnormalities and anticonvulsant drugs. Proc. R. Soc. Med., 63: 48-9, 1970.

23. MELNICK, $M$. et al. Cleft lip \pm cleft palate: an overview of the literature and an analysis of danish cases born between 1941 and 1968. Am. J. Med. Genet., 6: 83-97, 1980.

24. MESKIN, L.H. \& PRUZANSKY, S. Validity of the birth cerificate in the epidemiologic assessment of facial clefts. J. Dent. Res., 46: 1456-9, 1967.

25. MILLARD, D.R. \& MCNEIL, K.A. The incidence of cleft lip and palate in Jamaica. Cleft Palate J., 2: 384-8, 1965.

26. MOLLER, P. Cleft lip and cleft palate in Iceland. Arch. Oral Biol., 10: 407-20, 1965.

27. MORRISON, G. et al. The incidence of cleft lip and palate in the
Western Cape. S. Afr. Med.J., 68: 576-7, 1985.

28. MYERS, R.A. et al. Cleft lip and cleft palate in North Carolina. N. C. Med.J., 38: 707-12, 1977

29. NAGEM Fo, H. et al. Contribuição para o estudo da prevalência das más formaçōes congênitas lábio-palatais na população escolar de Bauru. Rev. Fac. Odont. Univ. S. Paulo, 6: 111$28,1968$.

30. NATSUME, N \& KAWAI, T. Incidence of cleft lip and cleft palate in 36,696 Japanese babies bom during 1983. Int. J. Oral. Maxillofac. Surg., 15: 565-8, 1986.

31. NATSUME, $N$ et al. The prevalence of cleft lip and cleft palate in Japanese : their birth prevalence in 40.304 infants born during 1982. Oral Surg. Oral Med. Oral Pathol, 63: 4213. 1987 .

32. NEEL, J.V. A study of major congenital defects in Japanese infants. Am. J. Hum. Genet., 10: 398-445, 1958.

33. RINTALA, A.E. Epidemiology of orafacial clefts in Finland: a review. Ann. Plast. Surg., 17: 456-9, 1987.

34. SAXÉN, I. Cleft lip and palate in Finland: parental histories, course of pregnancy and selected environmental factors. Int. J. Epidemiol., 3: 263-70, 1974.

35. SAXÉN, I. Associations between oral clefts and drugs taken during preganancy. Int.J. Epidemiol., 4: 37-44, 1975.

36. SAXÉN, I. Etiological variables in oral clefts.Proc. Finn. Dent. Soc., 71 (Suppl. 3): 1-40, 1975.

37. SCHLESSELMAN, J.J. Case-control studies: design conduct, analysis. New York, Oxford. University Press. 1982 (Monographs in Epidemiology and Biostatistics).

38. SESGIN, M.Z. \& STARK, R.B. The incidence of congenital defects. Plast. Reconstr. Surg., 27: 261-6, 1961.

39. SHAPIRO, S. et al. Anticonvulsants and parental epilepsy in the development of birth defects. Lancet, 1 (7954): 272-5, 1976.

40. SLONE, D. et al. Maternal drug exposure and fetal abnomalities. Material and Methods. Clin. Pharmacol. Ther., 14:648-53, 1973.

41. SMITHELLS, R.W. Environmental teratogens in man.Br.Med. Bull., 32: 27-33, 1976.

42. SOUZA, J.M.P. de \& BENICIO, M.H.D'.A. Análise multivariada: um exemplo usando modelo log-linear. Rev. Saíde Príblica, 19: 263-9, 1985.

43. SOUZA, J.M.P. de et al. Estudo da morbidade e da mortalidade perinatal em maternidade. III - Anomalias congênitas em nascidos vivos. Rev. Saíde Pública, 21: 5-12, 1987.

44. TANAKA, T. A clinical, genetic and epidemiologic study on cleft lip and/or cleft palate. Jap. J. Hum. Genet. , 16: 278308, 1972.

45. THOMPSON, J.S. \& THOMPSON, M.W. Genética médica. $2^{3}$ ed. Rio de Janeiro, Atheneu, 1976.

46. WEHRUNG, D.A. \& HAY, S. A study of seasonal incidence of congenital malformations in the United States. Br.J.Prev. Soc. Med., 24: 24-32, 1970.

Recebido para publicação ent 8.10.1993 Aprovado para publicação em 29.4.1994 